\title{
THE EFFECT OF AGE AND ILLUMINATION UPON VISUAL PERFORMANCE WITH CLOSE SIGHTS
}

\author{
BY \\ H. C. Weston

\section{INTRODUCTION}

THIs paper is based upon the results of an experimental investigation, in which twelve subjects performed a series of special visual tasks involving the perception of fairly small detail. Each task in the series was performed at each of six values of illumination which were so chosen as to form a geometric series. The lowest value was 0.5 lumen per square foot (i.e., 0.5 foot-candle), and the highest was approximately $5001 \mathrm{~m} / \mathrm{ft}^{2}$, so that the range covered all values likely to be used in practice for comparable tasks done either with natural or artificial lighting. The brightness of the visible environment (panoramic brightness) was varied pari passu with that of the local field of attention, so as to maintain a ratio of the former to the latter of not less than 0.5 .

As it was not the object of the experiments to obtain data relating to the variation of visual performance with age, the subjects were not chosen accordingly. Subsequently, however, it was found that they were distributed by age in five consecutive quinquenniads covering, approximately, the middle third of life. Hence, the data obtained relate to five small groups, in three of which there were two subjects, while in each of the other two groups there were three subjects. An unusual and, for the present purpose, a very fortunate circumstance, is that similar visual performance tests were done under similar illuminations by the same subjects at times separated by an interval of five years. So, it is possible not only to compare the performances of the five different age groups, but also to show the effect of a five year difference of age upon the performance of each group.

\section{Refraction and Acuity of Subjects}

All the subjects are experienced observers, being members of the scientific staff of the National Physical Laboratory. Prior to the second series of experiments, they were examined by Mr. Graeme Talbot, F.R.C.S., and new glasses were prescribed as required (see Table I).

All the subjects read J.I. with their glasses. One is a fairly high myope, but the others are only slightly ametropic. Group 
acuity does not appear to be related to group age and, in fact, the clinical findings show no important difference of acuity among the group.

\begin{tabular}{|c|c|c|c|c|}
\hline $\begin{array}{l}\text { Age } \\
\text { Group } \\
\text { at Date } \\
\text { of } \\
\text { refraction }\end{array}$ & Subject & R. eye & L. eye & Phoria at near \\
\hline \multirow{2}{*}{$24-28$} & 1 & $\frac{-0.25}{-0.25 \cdot 175^{\circ}}=6 / 5$ & $\frac{-0.25}{-0.50 \cdot 170^{\circ}}=6 / 5$ & 0 \\
\hline & 2. & $\frac{-2.5}{-0.5 \cdot 50^{\circ}}=6 / 6$ & $\frac{-2.5}{-0.5 \cdot 30^{\circ}}=6 / 6$ & $\begin{array}{c}4 \\
\text { ESO }\end{array}$ \\
\hline \multirow{3}{*}{$29-33$} & 3 & $\frac{-0.5}{+0.50 .95^{\circ}}=6 / 6$ & $\frac{-0.5}{+0.7560^{\circ}}=6 / 12$ & ESO \\
\hline & 4 & $\frac{0}{+0.2595^{\circ}}=6 / 6$ & $\frac{0}{+0.2580^{\circ}}=6 / 6$ & 0 \\
\hline & 5 & $\frac{0}{-2 \cdot 50 \cdot 10^{\circ}}$ & $\frac{-0.25}{-1.75 \cdot 173^{\circ}}$ & 0 \\
\hline \multirow{2}{*}{$34-38$} & 6 & $\frac{-1 \cdot 25}{-0.50 \cdot 160^{\circ}}=6 / 5$ & $\frac{-1.0}{-0.5 .55^{\circ}}=6 / 5$ & $\stackrel{4}{E^{S O}}$ \\
\hline & 7 & $\frac{+3.0}{+0.75 .83^{\circ}}=6 / 5$ & $\frac{+0.25}{-}$ & $\stackrel{2}{\text { EXO }}$ \\
\hline \multirow{3}{*}{$39-43$} & 8 & $\begin{array}{r}\frac{-0.25}{+1.0 .105^{\circ}}=6 / 5 \\
\text { NEAR ADD. }\end{array}$ & $\begin{array}{l}- \\
+0.5 .90^{\circ} \\
+0.5\end{array}$ & EX \\
\hline & 9 & $\begin{array}{c}- \\
+0.5 \cdot 10^{\circ} \\
\text { NEAR ADD }\end{array}$ & $\frac{-0.25}{+0.5 \cdot 145^{\circ}}=6 / 6$ & 0 \\
\hline & 10 & $\frac{-1.0}{-0.570^{\circ}}=6 / 6$ & $\frac{-1.25}{-0.25}, 35^{\circ}=6 / 6$ & 0 \\
\hline \multirow{2}{*}{$44-48$} & 11 & $\frac{-0.25}{+0.5 \cdot 25^{\circ}}=6 / 5$ & $\frac{-0.25}{+0.50 \cdot 110^{\circ}}=6 / 6$ & $\stackrel{4}{\operatorname{EXO}}$ \\
\hline & 12 & $\begin{array}{l}\frac{-9.5}{-1.0 .105^{\circ}}=6 / 6 \\
\quad \text { NEAR ADD. }\end{array}$ & $\frac{-12.5}{-0.5 \cdot 10^{\circ}}$ & EXo \\
\hline
\end{tabular}




\section{Measurement of Visual Performance}

In this paper visual performance means the rate of accomplishing a specific visual task, e.g., the discrimination of visual objects having a prescribed characteristic, when these objects are presented along with others in an objective field of sight whose angular size is typical of " near work." The test-objects employed were Landolt rings, arranged as shown in Fig. 1. The rings differ only in the orientation of the gap, eight positions of the latter being used. The visual task consisted in finding all the rings

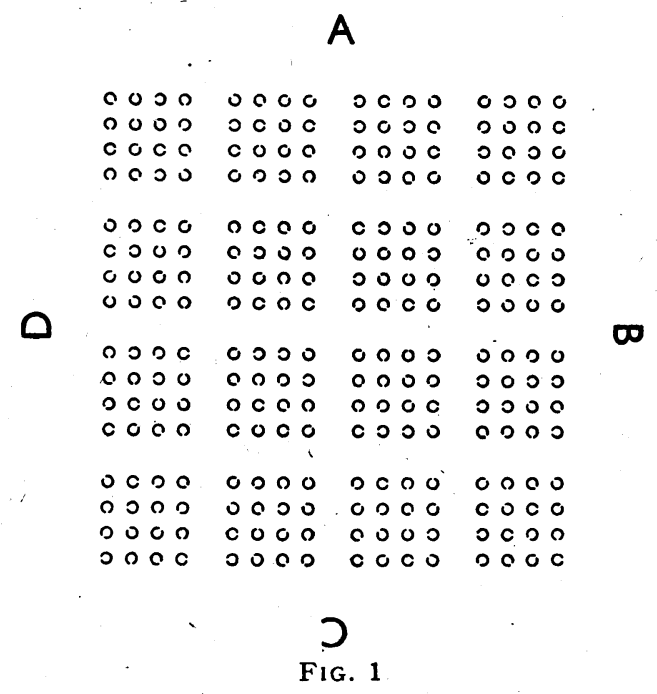

having the gap in one prescribed position, and so it involved some scrutiny of every ring present in " the sight." The severity of the visual task was varied by using three sizes of ring, such that, at ordinary reading distance, the visual angles subtended by the gaps were, respectively, $1.5,3$ and 4.5 minutes of arc. Of these sizes, the intermediate one corresponds approximately to the size of detail in the news columns of the daily press.

These visual tasks may be compared with practical tasks such as proof-reading, reading numerals, and many inspection tasks done in factories, offices, schools, etc. Clearly the rate of performance can only be ascertained by measuring the time taken by viewer to complete the task. But, as some reaction is necessary for the purpose of indicating that the objects of search have been recognized, the total time taken to deal with each test sight must include this reaction or motor response time. The two components of the time per test, that is, the visual discrimination time and the 
indicator action time, cannot be separately measured; hence, an exact determination of the discrimination time is impossible, though, by a suitable technique, it can be ascertained to a fairly close approximation. In these tests the method adopted was as follows. The subjects were asked to indicate the prescribed rings by cancelling them with a stroke of the pencil. They were first given a test sheet on which all the prescribed rings were made extremely conspicuous by being filled in with red ink, thus converting them to red spots. By this means the search time was minimised, the time taken by the subject to work through the test material being almost entirely time occupied in manual action. The test was then given in the ordinary way, so that the prescribed objects had to be found by discerning the gaps in the rings and their orientation. The time required to work through the test material in this way was measured, so that the time per correct ring cancelled could be found. From this was deducted the time required for the act of cancellation, as found from the red-spot test. The net time thus obtained is the closest practicable approximation to the actual discrimination time. This has been corrected when necessary, so as to allow for the fact that the subjects sometimes overlooked some of the rings which should have been cancelled, that is to say, it has been divided by an accuracy factor so as to obtain the time which would have been required for a perfectly accurate performance. The reciprocal of this corrected value is the index of speed of discrimination used in this paper.

\section{Results Obtained}

The individual performance values obtained at each illumination, and with each visual task, have been averaged for each age group. From these averages graphs were prepared showing the variation of performance with illumination. As would be expected, owing to the small number of observations available for each age group at any one illumination and size of test-object, all the points plotted did not lie upon smooth curves. Allowing, however, for unmistakably exceptional results, smooth curves were fitted to the plots, and the performance values derived from these curves are those which have been used in preparing Figs. 2, 3, and 4. In this connection it should be said that the smoothing of the actual observations, while it removes irregularities which are unlikely to have appeared had more data been available for analysis, does not effect the main conclusions to be drawn from these experiments; that is to say, the invariable decline of visual performance with age which is exhibited by the diagrams is found also, and on the average to the same extent, by comparison of the-raw data.

Referring now to Fig 2, it can be seen that the points plotted 


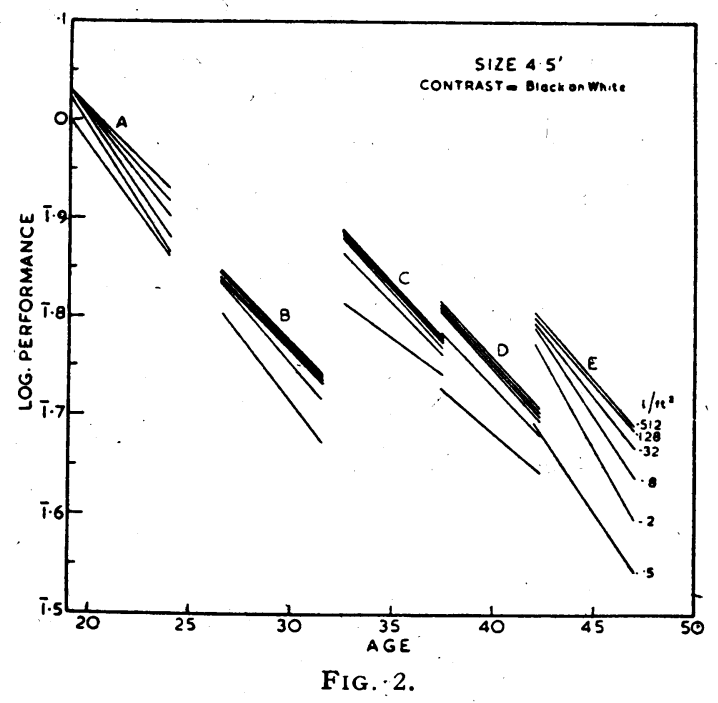

refer to ages ranging from 19 to 47 years, and to the logarithms of the value of visual performance. By plotting log. performance alt the results are shown on a relative basis, that is to say, the difference on the vertical scale between any two values, is proportional to the ratio of those values, and this ratio can readily be found, if desired, from tables of antilogarithms. For example, the scalar difference between the value plotted for group $E$ at $321 \mathrm{~m} / \mathrm{ft}^{2}$ and age 47 years, and that plotted for group $\mathrm{A}$ at the same illumination and age 24 years is $0 \cdot 23$, which means that E's performance is only equal to 59 per cent. of A's. Similarly, it can be found that A's performance at $0.51 \mathrm{~m} / \mathrm{ft}^{2}$ and age 24 , is about equal to 85 per cent. of the performance achieved by the same group at the same age when the illumination was increased to $500 \mathrm{~lm} / \mathrm{ft}^{2}$.

In Fig. 2 all the lines joining pairs of points have a downward slope, so that it is clear that an advance in age of 5 years is accompanied by a decline in performance in the case of each group, and with all the values of illumination. Consistent with this finding is the obvious decline from group to group as the group-age advances. The general trend of the results might be shown by drawing a straight line across the diagram in such a way that approximately an equal number of all the points plotted lies above and below the line. If this were done it would be found that the downward slope of this line is less steep than that of the lines drawn for each separate age group, so that the annual rate of decline which might be estimated in this way would be lower than 
the rate found from the 5-year difference of performance actually observed for each group. One reason for this apparent discrepancy is that the relative levels of the group families of curves certainly depend partly upon group differences of visual ability which, no doubt, existed when all the subjects of these experiments were of the same age. As to this, it is clear that no direct evidence can be had, but the disposition of the families of curves, and particularly the obvious displacement of the B group family, leaves little room for doubt that the different groups would have been found unequal in efficiency could their performance have been measured when they were of equal age. Thus, the decline in performance from one age group to next does not indicate the true rate of decline with advancing age. The true rate can only be found from chronologically different performances of the same individuals or groups, such as are here presented. But, since these chronologically different performances refer only to a 5 -year change of age, they do not enable the annual rate of decline to be found for the whole middle third of life, nor do they show whether the rate is constant or variable during this period. On these points, however, the results shown in Fig. 2 are suggestive. It can be seen that, except at the lower values of illumination, the slope of the curves is much the same for each age group, and so, for the particular visual task considered, it would appear that the rate of decline does not vary much from year to year, though it tends to increase during the last quinquennium, i.e., between the ages of 42 and 47 . On the average, it is of the order of 5 per cent. per annum with moderate to high values of illumination.

The illumination relating to each performance curve is shown in Fig. 2. It is evident that varying the illumination of the test material has a more marked effect upon visual performance as age advances and, in the case of group E, the spread of the family of curves is twice as wide as it is for group A. It may be noted that, at the age of 45 , the performance achieved with an illumination of $5001 \mathrm{~m} / \mathrm{ft}^{2}$ is not as good as that achieved at the age of 25 years, with an illumination of only $0.5 \mathrm{~lm} / \mathrm{ft}^{2}$. Moreover, after the lapse of 5 years, no group gives the same performance at the highest illumination as it formerly did at the lowest illumination. Although this result is surprising, and is not consistent with the results obtained with smaller test-objects, it seems clear that any considerable advance in age cannot be fully compensated by any reasonable adjustment of the illumination level for visual tasks of this kind.

Turning now to Fig. 3, it will be seen that the decline from group to group is somewhat steeper than it is with the larger testobject dealt with in Fig. 2. But the annual rate of decline, as indicated by the different families of curves, is much the same. Here, however, in the case of the older subjects-groups C, D, 
EFFect of Age and Illumination Upon Visual

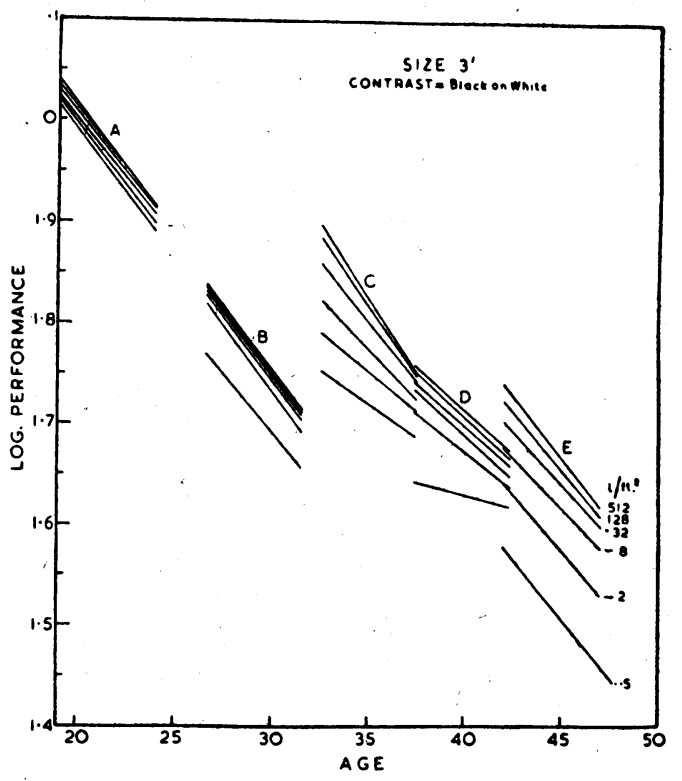

FIG. 3.

and $\mathrm{E}$-an illumination of $81 \mathrm{~m} / \mathrm{ft}^{2}$ gives a performance similar to that achieved 5 years earlier by the same subjects with an illumination of $0.51 \mathrm{~m} / \mathrm{ft}^{2}$; while $5001 \mathrm{~m} / \mathrm{ft}^{2}$ gives a performance similar to that formerly given by $21 \mathrm{~m} / \mathrm{ft}^{2}$, though, again, it is evident that high illumination does not fully compensate for any considerable advance in age.

Fig. 4 shows the results obtained for the most difficult visual task. As might be expected, the greatest decline from group to group is here exhibited, and the average annual rate of decline throughout the age span considered is more than 7 per cent. The spread of the families of curves relating to age groups $B, C, D$, and $E$ is much wider than is the case with the easier visual tasks, thus showing that the performance of all the subjects in these age groups, i.e., those whose age is 25 years or more, is much affected by the level of illumination, particularly when this falls below about $301 \mathrm{~m} / \mathrm{ft}^{2}$. Actually, a change from the lowest to the highest illumination tried increases the performance of the youngest subjects only by 18 per cent., but it increases the performance of the oldest subjects fourfold. It is also of interest to note that, with the lowest illumination and the finest visual task, the performance of subjects aged 24 years is just as good as that of subjects aged 47 years with the easiest visual task, and the highest illumination (compare Figs. 2 and 4 ). 


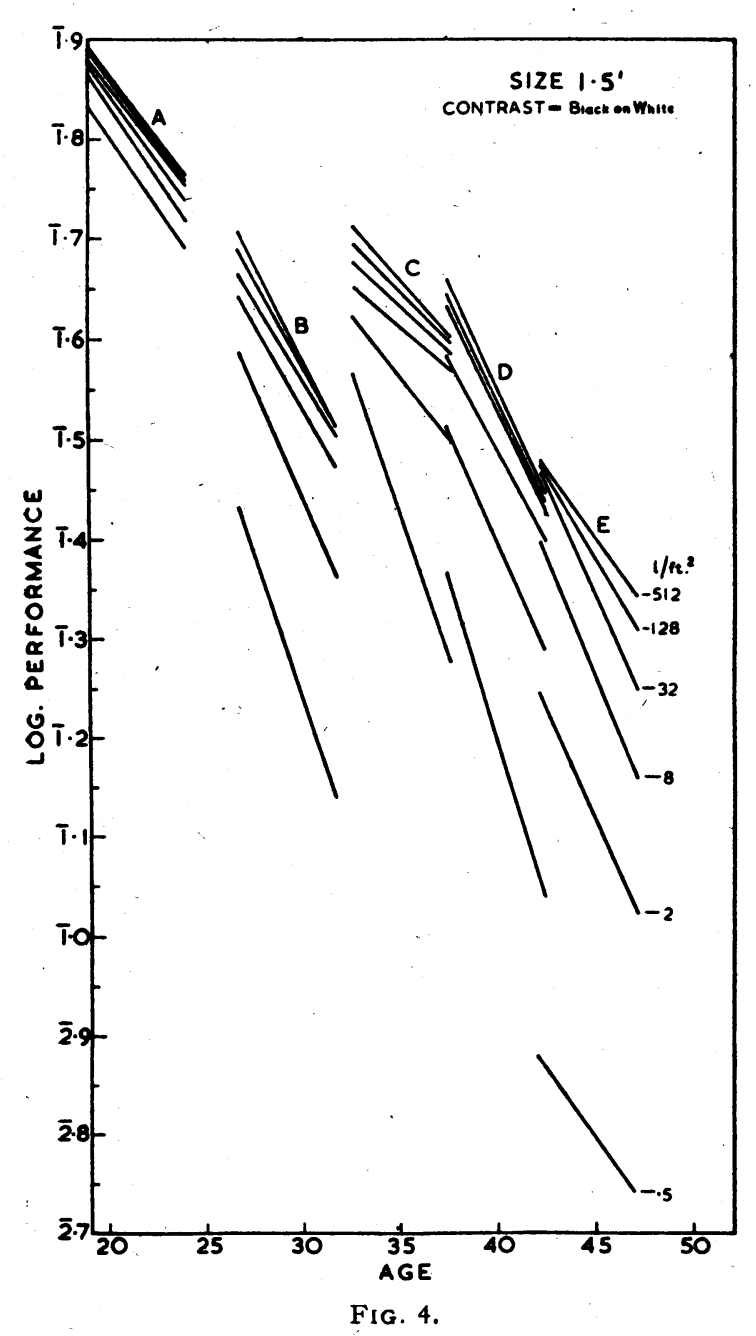

\section{Conclusion}

It should be clearly understood that the decline shown by the results of the experiments discussed does not refer to the resolving power of the eyes, but to the quickness of perception, that is, to a function of vision which is not evaluated by clinical tests. Even so, the extent of the decline, and its early onset, may seem surprising, and perhaps depressing. It must be remembered, however, that most everyday visual tasks do not involve continuous scrutiny of small detail, and so the performance of them will not generally be so much affected in the advance to "middle-age" 
as is the performance of the visual tasks considered in this paper. Nevertheless, visual efficiency appears to come to its maximum in early adulthood, and, thereafter, to fall off at a greater rate than we realise. This is in keeping with other findings concerning ageing. For example, Bouma ${ }^{1}$ has shown that contrast sensitivity is a function of age, and that it diminishes from the mid-twenties onwards; Mann and Sharpley ${ }^{2}$ have found there is a tendency for the rod field of the dark-adapted eye to contract with advancing age, this tendency becoming apparent at about the age of 30 years; and it has been shown that manual motility begins to decline in the late twenties ${ }^{3}$. Probably many accidents should be attributed to the slowing down of vision as age advances, and it is well known that dissatisfaction with standards of artificial lighting commonly found in workplaces is most prevalent among middleaged and elderly workers. The general tendency for visual performance to decline from a fairly early age, which, in spite of the few subjects studied, is so consistently shown by the results presented in this paper, is certainly subject to individual exceptions. Indeed, individually, some of the subjects of these experiments showed different rates of decline, and probably began their decline at different, though not widely different, ages.

\section{REFERENCES}

1. Bouma.-Philips Technical Review, Vol. IX, No. 5, p. 151, 1947.

2. Mann and Sharpley.- Jl. Physiol, Vol. CVI, pp. 301-304, 1947.

3. Miles.--In Cowdry's “ Problems of Ageing," pp. 758-759, 1931.

\section{SIDELIGHTS ON THE INFERIOR OBLIQUE MUSCLE}

BY

\section{J. Ringland ANDERSON}

MELBOURNE

\section{ANATOMICAL $\cdot$ Features}

The relationships of this muscle to the inferior rectus and to the capsule of Tenon and neighbouring orbital fascia require emphasis. These are assuming greater importance as the inferior oblique muscle is directly or indirectly concerned with an increasing number of surgical procedures. The connection between the sheaths of the two inferior muscles and 'Tenon's capsule restricts the effect of division of the inferior oblique muscle. The results of such an operation may vary with the intimacy of such connections and the strength of the check ligaments. 